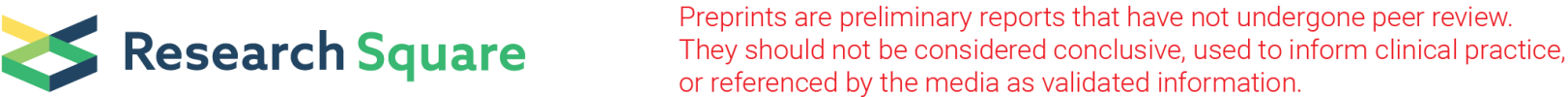

\section{Haemophilus Influenzae One Day in Denmark: Prevalence, Circulating Clones, and Dismal Resistance to Aminopenicillins}

niels nørskov-lauritsen ( $\nabla$ niels.norskov-lauritsen@rsyd.dk)

Department of clinical microbiology, Odense University Hospital https://orcid.org/0000-0001-5520106X

\section{Nanna Pedersen}

Aarhus University Hospital: Aarhus Universitetshospital

Janni Lam

Data integration and analysis, State serum institute

\section{Hans Nielsen}

Aalborg University Hospital: Aalborg Universitetshospital

\section{Carl Kobel}

Aarhus Universitetshospital

\section{Dennis Hansen}

Copenhagen university hospital Herlev

\section{Research Article}

Keywords: Point prevalence, phylogenetic group I, penicillin-binding protein 3, competence

Posted Date: February 24th, 2021

DOl: https://doi.org/10.21203/rs.3.rs-234959/v1

License: (c) (i) This work is licensed under a Creative Commons Attribution 4.0 International License.

Read Full License

Version of Record: A version of this preprint was published at European Journal of Clinical Microbiology \& Infectious Diseases on April 23rd, 2021. See the published version at https://doi.org/10.1007/s10096021-04247-w. 


\section{Abstract}

\section{Purpose}

Haemophilus influenzae is a common cause of mucosal infections that warrants accurate surveillance. We aimed to assess the prevalence of the species in clinical specimens, and characterise population structure and resistance to aminopenicillins by whole genome sequencing.

\section{Methods}

We assessed the point prevalence by entering the database records of one day in Denmark, and examined the genome sequences of nationwide, collected isolates from the same day.

Results

The prevalence of $\mathrm{H}$. influenzae in clinical samples on the $10^{\text {th }}$ of January 2018 was 1.78 per 100,000 person-days (all samples), and 2.47 per 1,000 hospital bed-days (hospital samples). Of 2,009 bacteria deemed clinically relevant and collected in a concerted action by the Danish departments of clinical microbiology, 62 (3.1\%) were $\mathrm{H}$. influenzae. All 62 isolates belonged to phylogenetic group I and were unencapsulated. Three strains from separate Danish regions had identical core genome sequences, but a small number of intergenic mutations testified to circulating clones, rather than individual cases of patient-to-patient transmission. The TEM-1 $\beta$-lactamase gene was present in 24 strains, while 13 strains were genetically categorised as ampicillin-resistant due to substitutions in penicillin-binding protein 3 ; shared patterns of amino acid substitutions in unrelated strains indicated putative lateral transfer of chromosomal resistance.

\section{Conclusions}

Circulating clones of $\mathrm{H}$. influenzae are frequent, and host factors, rather than direct transmission of epidemic strains, may be the primary cause of infection. The bleak presence of ampicillin resistance revealed by sequencing of point prevalence strains underscores the necessity for close examination of testing methods.

\section{Introduction}

Haemophilius influenzae is a small Gram-negative bacterium that is part of the normal microbiota of the human upper respiratory tract. $H$. influenzae can cause pneumonia, otitis media, sinusitis and conjunctivitis, and more rarely, invasive disease [1-3]. H. influenzae type b (Hib) conjugate vaccine effectively prevents childhood meningitis and other invasive infections caused by serotype b capsulate strains, and replacement with other capsulate types has been limited [3-5]. However, unencapsulated $H$. influenzae is a major cause of mucosal infections [6], and the global burden is estimated to represent $55-95 \%$ of cases of otitis media in children, $44-68 \%$ of cases of childhood conjunctivitis, and more than $90 \%$ of acute exacerbation of chronic obstructive pulmonary disease [1 and references therein]. 
H. influenzae with resistance to ampicillin and amoxicillin was reported in the 1970 s $[7,8]$. Two distinct $\beta$ lactamases have been identified, ROB-1 and TEM-1, which confer high-level resistance to ampicillin and other aminopenicillins, but are unable to degrade cephalosporins at a significant rate [9]. Extended spectrum $\beta$-lactamases have not been documented for $H$. influenzae. A major concern relates to amino acid substitutions in the transpeptidase region of penicillin-binding protein (PBP) 3 , which is the sole indispensable PBP of the species [10]. $\beta$-lactamase-negative ampicillin-resistant (BLNAR) strains with mutational resistance are usually grouped according to decisive substitutions and antimicrobial spectrum, where group I and II confer resistance to aminopenicillins only, while the target range of group III includes cephalosporins [11, 12]. Resistance to third generation cephalosporins in $H$. influenzae have only slowly emerged in Europe [12-14], while it is prevalent in Southeast Asia [15, 16]. Restricted use of oral cephalosporins probably constrains the emergence of resistance in particular global areas, but development of broad antimicrobial resistance in $H$. influenzae may not be preventable. In 2017, the World Health Organization published a list of antibiotic-resistant pathogens for which new and effective antibiotics are urgently needed [17]. Ampicillin-resistant $H$. influenzae was included in the same priority tier as penicillin-resistant Streptococcus pneumoniae.

Previously, we described a nationwide point-prevalence survey of human bacterial pathogens in Denmark. On the 10th of January 2018, 2,009 bacterial isolates deemed clinically relevant were collected in a joint operation by all departments of clinical microbiology (DCMs) [18]. Sixty-two (3.1\%) of the collected bacteria were $H$. influenzae. Here, we describe the epidemiology, genome characteristics, and $\beta$-lactam resistance of $H$. influenzae identified in clinical specimens on a single day in Denmark.

\section{Materials And Methods}

\section{Specimen reports, collection and sequencing of strains}

The Danish microbiology database, MiBa, receives copies of specimen reports from all Danish DCMs [19]. We extracted reports of $H$. influenzae released on the 10th of January 2018 with information of sample type, reported antimicrobial resistance, affiliation of requisitioning physicians (hospital department or general practise), and reporting DCM. Original sample number and person identifiable data were blinded to the study.

Collection of clinically relevant bacteria from all Danish DCMs on the 10th of January $2018(N=2,009)$, genome sequencing using the Illumina NextSeq 500 platform, and distribution of species have been described [18]. The definition of "clinical relevance" was at the discretion of the responsible DCM, however, the general criterion for inclusion was the report of identified bacterial species plus assessment of antimicrobial resistance. Original sample number and person identifiable data were blinded to the study.

Whole genome sequence (WGS) analysis of $\mathrm{H}$. influenzae 
Sixty-two bacteria collected on the 10th of January 2018 were H. influenzae, confirmed by K-mer alignment with ribosomal proteins at the pubMLST database [18]. Raw reads from these 62 strains were re-assembled and initially compared using the nullarborv2 reads-to-report package [20]. Reads were trimmed with trimmomatic v0.39 [21], and assembled with shovill v1.0.9 for skesa v2.3.0 [22]. Annotation with Prokka, and identification by Roary of core genes present in all strains were performed as previously described [23]. Concatenated core genes were aligned across all samples and single nucleotide polymorphism (SNP) distances were counted with snp-dists v0.7.0. To include intergenic mutations, SNPs were also called by mapping of reads to closed circular DNA of strain Rd KW20 (NC_000907), using snippy v4.4.3; the read mapping-method gave rise to 129,997 SNPs or $7.1 \%$ of the Rd KW20 genome. MLST sequence types were identified with $\mathrm{mlst}$ v2.18.0 (https://github.com/tseemann/mlst), and the Haemophilus influenzae database (PubMLST.org) updated February 2020. Resistance genes were screened for by use of abricate v0.9.8 [https://github.com/tseemann/abricate] assessing the Bacterial Antimicrobial Resistance Reference Gene Database (https://www.ncbi.nlm.nih.gov/pathogens/isolates\#/refgene/).

WGSs (24-56 contigs except strain 0549 that gave rise to 148 contigs) were uploaded to Pathosystems Resource Integration Center (PATRIC) [24], and genes for $\beta$-lactam mutational resistance (PBP3), fluoroquinolone resistance (GyrA. GyrB, ParC, ParE), capsule polysaccharide exporter protein (BexA), plus 17 genes indispensable for natural competence [25] were identified by BLAST, downloaded, and compared using MEGA X [26].

\section{Accession numbers}

Allelic profiles of four strains with new MLST sequence types have been uploaded to PubMLST.org and assigned sequence types 2329-30 and 2332-3; WGSs of the same four strains have been deposited in GenBank under accession numbers JAECZV000000000-JAECZY000000000. Raw sequence data of the entire ODiD 2018 collaboration have been submitted to the European Nucleotide Archive under study accession no. PRJEB37711 [18].

\section{Results}

\section{Data reported to the Danish microbiology database}

One hundred and three specimens from 103 patients with growth of $H$. influenzae were reported by the Danish DCMs on the 10th of January 2018, corresponding to a detection rate of 1.78 per 100,000 persondays. Denmark is divided into five regions, and regional detection rates varied between 1.37 and 3.57, median rate 1.72. Thirty-four samples were from hospitals, corresponding to a detection rate in clinical specimens of 2.47 per 1,000 hospital bed-days (range 1.04-5.37, median 1.95) (Table 1). Samples originated primarily from suspected cases of conjunctivitis ( $N=48 ; 47$ from practising physicians) and from the respiratory tract $(\mathrm{N}=39 ; 32$ from hospital departments including three bronchial samples). Other anatomical locations were ear (10) and nose (six). 
Table 1

Prevalence of $H$. influenzae in clinical specimens in Demark

\begin{tabular}{|c|c|c|c|c|c|c|}
\hline $\begin{array}{l}\text { Region } \\
\text { (No of } \\
\text { DCMs) }\end{array}$ & Population & $\begin{array}{l}\text { Samples with } H \text {. } \\
\text { influenzae on } \\
\text { January 10th }\end{array}$ & $\begin{array}{l}\text { Detection rate } \\
\text { per } 100.000 \\
\text { person-days }\end{array}$ & $\begin{array}{l}\text { Samples } \\
\text { from } \\
\text { Hospital }\end{array}$ & $\begin{array}{l}\text { Hospital } \\
\text { beds }\end{array}$ & $\begin{array}{l}\text { Detection } \\
\text { rate per } \\
1,000 \\
\text { hospital } \\
\text { beds }\end{array}$ \\
\hline $\begin{array}{l}\text { North } \\
\text { Denmark } \\
\text { Region (1) }\end{array}$ & 589,000 & 21 & 3.57 & 7 & 1,303 & 5.37 \\
\hline $\begin{array}{l}\text { Central } \\
\text { Denmark } \\
\text { Region (1) }\end{array}$ & $1,313,500$ & 21 & 1.60 & 10 & 2,788 & 3.59 \\
\hline $\begin{array}{l}\text { Region of } \\
\text { Southern } \\
\text { Denmark (4) }\end{array}$ & $1,220,500$ & 21 & 1.72 & 5 & 2,602 & 1.92 \\
\hline $\begin{array}{l}\text { Region } \\
\text { Zealand (1) }\end{array}$ & 835,000 & 15 & 1.80 & 2 & 1,930 & 1.04 \\
\hline $\begin{array}{l}\text { Capital } \\
\text { Region of } \\
\text { Denmark (3) }\end{array}$ & $1,822,000$ & 25 & 1.37 & 10 & 5,123 & 1.95 \\
\hline All Denmark & $5,780,000$ & 103 & 1.78 & 34 & 13,746 & 2.47 \\
\hline
\end{tabular}

Antimicrobial susceptibility testing (AST) was not reported for 26 samples ( 23 conjunctiva, 2 nose and 1 ear). One DCM reported absence of $\beta$-lactamase for eight samples (three ear, three conjunctiva, and two nose) and stated that "penicillin derivatives will normally be active". With respect to conjunctival samples, two DCMs reported AST of chloramphenicol and ciprofloxacin (12 samples), or fusidic acid and tobramycin (10 samples), respectively. AST of $\beta$-lactams was reported for all respiratory tract cultures plus six ear- and two nasal cultures. Two departments did not record zone diameters, but reported AST from four respiratory samples (including three from cystic fibrosis patients) based on visual estimation of zones. As determined by reported AST of 47 strains from 10 DCMs, 30 strains (64\%) were ampicillinsusceptible, eight (17\%) were resistant to ampicillin but susceptible to amoxicillin-clavulanate, and 9 (19\%) were resistant to amoxicillin-clavulanate.

\section{WGS: Genome relatedness and circulating clones}

Sixty-two of 103 reported strains of $H$. influenzae were collected for genetic characterisation. Figure 1 depicts genome relatedness and lists regional origin, specimen type and selected WGS characteristics. $H$. influenzae consists of two distinct, phylogenetic groups, where group II comprises serotypes e and f, plus other rarely detected lineages [27]. The 62 isolates of the point prevalence study all belonged to phylogenetic group I, and none of them encoded a capsulation operon (often designated "NTHi", nontypeable Haemophilus influenzae). Multilocus sequence typing (MLST) revealed a major cluster of sequence type (ST) 103, plus some smaller clusters (ST11, ST155, ST160, ST367, ST388 and ST652). 
Strains of the same ST frequently originated from different regions of Denmark. Extending the size of compared DNA, from seven gene fragments (MLST) to 1,166 concatenated core genes, disclosed some SNPs differentiating strains of the same ST, but three ST103 strains $(0575,0802$ and 0876) from three separate regions contained identical core genomes. If the genomic comparison was limited to the eight closely related ST103 strains, the number of core genes increased from 1,166 to 1,791 (1.137 to $1.673 \mathrm{Mb}$ ), but SNPs differentiating strain 0575,0802 and 0876 were still absent. Only inclusion of intergenic regions, by mapping of reads to the closed circular reference genome RD KW20, was able to disclose 35-58 SNPs separating the three strains.

Figure 2 shows pairwise SNP distances among 8 closely related ST103 strains (28 pairs) as revealed by comparison of core genes and by read mapping. The two methods were not fully coherent, as observed for the three strains with identical core genes, as well as other unexpected differences. In the complete collection of 62 strains, seven pairs were related below a breakpoint of 40 SNPs by read mapping, and two of them differed by only seven (0648 vs 0778, ST155) and 16 SNPs (0803 vs 0846, ST103). The human hosts of the former pair were separated by a wide geographic distance, and although the number of SNPs was low, pairwise Roary-quantification of shared genes in the two genomes sequestered 68 of 1,718 annotated genes to presence in only one of the two strains. The latter pair originated from the same region albeit from separate wards, and pairwise comparison revealed a relatively large number of shell genes (59 of 1881 annotated genes were present in only one of two strains). A third pair (0536 and 1351, ST107) was separated by 31 SNPs by read mapping, but encompassed a comprehensive core, with only two of 1729 annotated genes being confined to the shell.

\section{WGS: Acquired and mutational resistance}

The TEM-1 $\beta$-lactamase gene (bla $\mathrm{TEM}-1)$ was present in 24 strains (39\%), whereas ROB-1 was absent. TEM-1 was associated with integrative and conjugative element ICEHin1056 in 22 strains, while two ST57 strains (0475 and 0476) encoded TEM-1 on small plasmids identical to pA1606 previously described from Denmark [28]. The coverage of the pA1606 contigs was 15 and 14 times the average sequence depth of contigs, respectively, demonstrating that pA1606 is a low copy number plasmid. For the other $\beta$-lactamase-positive strains, the coverage of TEM-1 did not differ from average genome sequence depth (mean ratio 1.03, range 0.72-1.24); thus, there was no evidence of extensive gene amplification of $b / a_{\mathrm{TEM}-1}$, which has been shown for piperacillin/tazobactam-resistant strains of Escherichia coli [29].

PBP3 was downloaded from 62 strains, and substitutions in the second half of the protein (which encompasses the transpeptidase region) were determined using strain Rd KW20 as reference. A total of 134 amino acid substitutions were disclosed at 18 positions (range 1-23) (Fig. 3). Substitution R517H (defining group I BLNAR) was not detected, but N526K was present in 13 strains (21\%), and these strains are therefore genetically categorised as ampicillin-resistant. Nine of the 13 strains were $\beta$-lactamase gene-negative, while four had simultaneous presence of $b / a_{\mathrm{TEM}-1}$ and may be designated beta-lactamasepositive ampicillin-clavulanic acid resistant (BLPACR); in the present context, the 13 strains are merged 
into PBP3 group II (Fig. 1). Seven strains encoded substitution M377I, close to the SSN motif at position $379-81$. M377I was included in the original definition of BLNAR group III that confer resistance to cephalosporins (M377I, S385T plus L389F; [11]), but the substitution has been omitted in recent updates of the scheme [12]. Two-thirds (82 of 121) of non-N526K substitutions in the second half of PBP3 occurred in PBP3 group II strains (Fig. 3, downward columns), with positions 547 as a noticeable exception (position 603 is outside the transpeptidase region). Inactivation of indispensable genes for natural competence was detected in nine strains (Fig. 1, column comp ${ }^{-}$), most often caused by single nucleotide mutations or deletions giving rise to premature stop codons. Truncation of essential competence genes showed a distinct association with wild-type PBP3 (eight of nine strains).

Sequence comparison of $\mathrm{fts}$ (encoding PBP3) is shown in Fig. 4. Thirteen strains with substitution N526K cluster in two groups, with 7 strains in cluster A and 3 strains in cluster B, plus three singletons ( $f t s /$ cluster designations are appended to PBP3 group II designations in Fig. 1). Cluster A strains encoded five substitutions in addition to N526K, namely D350N, M377I, A502V, V547I and N569S (the exception being strain 0473 with $D \rightarrow S$ at position 350). Cluster B strains encoded D350N, G490E and A530S, in addition to N526K. The singletons designated C1-C3 encoded two, three or one additional substitution (A530S plus V547I; D350N, A502T plus N569C; and R502V, respectively). The B cluster of $\mathrm{fts} /$ was only observed among closely related strains (ST107 and a new ST, Fig. 1, bottom), while fts/ A cluster strains are dispersed in the genome dendrogram.

Sixteen positions in GyrA. GyrB, ParC and/or ParE are associated with fluoroquinolone resistance in $H$. influenzae [30]. Amino acid substitutions at these positions were not detected.

\section{Discussion}

Two thousand and nine bacterial strains, reported on the 10th of January 2018 and deemed clinically relevant by the local DCM in charge, were collected for genetic characterisation. Sixty-two (3.1\%) were $H$. influenzae. Selection of strains was at the discretion of the responsible DCM, and criteria varied. Based on electronic reporting, the true prevalence on the 10 th of January was 103 isolates of $H$. influenzae in 103 samples from 103 patients, corresponding to 1.78 per 100,000 person-days (all samples), or 2.47 per 1,000 hospital bed-days (hospital samples). One Danish region reported 15 specimens with $H$. influenzae and submitted 15 strains for characterisation; the nadir was 21 reported vs four collected. However, 62 genetically characterised isolates constitute a solid and credible fraction of 103 point prevalence strains.

All 62 sequenced isolates belonged to phylogenetic group I and were unencapsulated. Phylogenetic characterisation of the species usually focuses on diversity, as well as strains from invasive infections $[27,31,32]$, and this aim may explain the overrepresentation of phylogenetic group II and the six capsular types, as well as outgroup strains, in published reports. The current depiction of the species underlines the importance of phylogenetic group I strains as commensals and in mucosal infections. 
The genomic characterisation disclosed nationwide, circulating clones. H. influenzae has rarely been subject to epidemiologic investigations, and the current study revealed some obstacles when genomic distances are interpreted in order to guide infection control. Due to inclusion of intergenic sequences, read mapping invariably generated larger, pairwise SNP distances than alignment of core genes. However, the "molecular clock" of the two bioinformatics tools was not congruent, as demonstrated by the discordance of the two methods to identify the most closely related genomes in the large cluster of ST103 strains (Fig. 2). Roary-estimation of shared genes of close neighbours did, in some cases, seclude annotated genes to presence in only one of the two strains. Uptake of genes can also reflect a molecular clock, but true assessment of genomic content will require closing of genomes, or use of long-read sequencing technology. The most significant or decisive bioinformatics tool for epidemiologic investigations has yet to be clarified.

Susceptibility to ampicillin was assessed by phenotype (growth in the presence of antimicrobial agent) for 47 strains, and by genotype (WGS) for 62 strains. Categorisation of resistance differed markedly, with ampicillin resistance rates of $36 \%$ and $53 \%$, respectively. A similar difference was reported from Japan, where $39 \%$ of upper respiratory tract isolates were non-susceptible to ampicillin (MIC $\geq 2 \mu \mathrm{g} / \mathrm{ml}$ ), while $65 \%$ were genetically $\beta$-lactamase-negative ampicillin-resistant (gBLNAR) [15]. Personal identifiers and original specimen numbers were blinded to this study, and the two samplings retrieved from the same prevalence material of 103 strains were not identical. Ampicillin-resistant strains may be overrepresented in conjunctival strains rarely subjected to AST, but it should be emphasised that the two definitions of resistance are not interchangeable. Mutational resistance comes with a wide range of MIC distribution, and the accepted precision of \pm 1 dilution for reference broth microdilution AST [33] complicates the interpretation when recorded MICs are close to the breakpoint. Partly to address the difficult identification of strains with mutational resistance, EUCAST has recently suggested that $H$. influenzae can only be categorised as susceptible to oral amoxicillin if an increased dosage of 750-1000 mg X 3 is used (EUCAST Clinical Breakpoint Tables v. 10.0). Finally, the unstable growth of fastidious organisms can affect the categorisation of phenotypic resistance. It is well known that methods and media have important consequences for detection of resistance in $\mathrm{H}$. influenzae [34]. General use of the EUCAST diskdiffusion method for categorisation of susceptibility to aminopenicillins has reduced the inter-laboratory inconsistency, but essential and categorical agreement with minimal inhibitory concentration determined by broth microdilution is still modest [35].

PBP3-substitution N526K was detected in 13 of 62 strains (21\%), which is twice the rate that was reported from one Danish region in 2007 [36]. The current strains showed a conspicuous accumulation of certain additional substitutions in the transpeptidase region of the protein. PBP3 substitutions identical to $\mathrm{fts} /$ clusters A and B were also common in Norway in 2007, being disclosed in 48 (43\%) and $17(15 \%)$ of 111 strains with the N526K substitution [37]. Although genetic comparison in that study was restricted to MLST, a similar tendency to congregation of $f t s /$ cluster B, and dispersion of $f t s /$ cluster A, was observed. This "linkage disequilibrium" between $\mathrm{fts} /$ cluster $\mathrm{A}$ and other core genes can arise by selection of particular mutations associated with survival in the presence of repeated exposure to antimicrobials, or by lateral transfer of an optimised fragment of the PBP3 gene. Inactivation of essential competence 
genes was preferentially observed in strains with wild-type PBP3, but the prevalence of truncated competence genes was too low to firmly disclose the origin of the gene fragment(s) encoding N526K. There is extensive variation in natural competence in $H$. influenzae [38], and other genes may markedly depress transformation without being essential for competence. Biologic assessment of competence in a random sample of clinical strains is required to elucidate the putative importance of lateral transfer for mutational resistance in $\mathrm{H}$. influenzae.

The point prevalence study has emphasised the significance of unencapsulated $H$. influenzae, which may be an under-recognised pathogen [1]. Circulating clones are frequent, but infections may be related to host factors, rather than direct transmission of epidemic strains. Revelation of aminopenicillin resistance in half of point prevalence strains by WGS must be addressed by meticulous AST, and confirms the concerns that have been raised by WHO.

\section{Declarations}

Acknowledgements Members of the ODiD Consortium are as follows: Ana Rita Rebelo, Valeria Bortolaia, Pimlapas Leekitcharoenphon and Frank Møller Aarestrup (Technical University of Denmark); Hans Linde Nielsen (DCM Aalborg); Svend Ellermann-Eriksen (DCM Aarhus); Turid Snekloth Søndergaard (DCM Sønderborg); John Eugenio Coia (DCM Esbjerg); Claus Østergaard (DCM Vejle); Michael Kemp (University of Southern Denmark); Bent Løwe Røder (DCM Slagelse); Niels Frimodt-Møller (DCM Rigshospitalet); Dennis Schrøder Hansen (DCM Herlev); Henrik Westh (DCM Hvidovre).

Authors' contribution The study was conceived and planned by NNL. JL retrieved data from MiBa, and CMK performed the major part of the bioinformatics analysis. NNL analysed data and wrote the first draft of the manuscript, which was revised critically for clinical content by NP, HLN and DSH. All authors approved the final version of the manuscript.

Data availability Accession numbers are given in the methods section. The dataset analysed as part of the current study is available from the corresponding author on reasonable request.

\section{Compliance with ethical standards}

Conflict of interest The authors declare that they have no conflict of interest.

Ethical approval and informed consent Not applicable to the present study. Reported culture of $H$. influenzae and collected bacterial strains are linked to specimen type and a regional DCM. Information cannot be linked to patient records or original sample number, and according to Danish law, approval from science ethics committees is not required.

Disclaimer The content of this manuscript is solely the responsibility of the authors and does not necessarily represent the official views of individual DCMs, the Danish Society of Clinical Microbiology, or the Danish Microbiology Database. 


\section{References}

1. Van Eldere J, Slack MPE, Ladhani S, Cripps AW (2014) Non-typeable Haemophilus influenzae, an under-recognised pathogen. Lancet Infect Dis 14:1281-1292. doi: 10.1016/S1473-3099(14)70734-0

2. Slack MPE (2015) A review of the role of Haemophilus influenzae in community-acquired pneumonia. Pneumonia 6:26-43. doi: 10.15172/pneu.2015.6/520

3. Heliodoro CIM, Bettencourt CR, Bajanca-Lavado MP, Portuguese Group for the Study of Haemophilus influenzae invasive infection (2020) Molecular epidemiology of invasive Haemophilus influenzae disease in Portugal: an update of the post-vaccine period, 2011-2018. Eur J Clin Microbiol Infect Dis 39:1471-1480. doi: 10.1007/s10096-020-03865-0

4. Gessner BD, Adegbola RA (2008) The impact of vaccines on pneumonia: key lessons from Haemophilus influenzae type b conjugate vaccines. Vaccine 26:B3-8. doi:

10.1016/j.vaccine.2008.04.013

5. Ulanova M (2020) Invasive Haemophilus influenzae serotype a disease in the Hib conjugate vaccine era: where are we going? Clin Infect Dis . doi: 10.1093/cid/ciaa868. Online ahead of print

6. Gallo MC, Kirkham C, Eng S, Bebawee RS, Kong Y, Pettigrew MM, Tettelin H, Murphy TF (2018) Changes in IgA Protease Expression Are Conferred by Changes in Genomes during Persistent Infection by Nontypeable Haemophilus influenzae in Chronic Obstructive Pulmonary Disease. Infect Immun 86:e00313-18. doi: 10.1128/IAl.00313-18

7. Nelson JD (1974) Editorial: Should ampicillin be abandoned for treatment of Haemophilus influenzae disease? JAMA 229:322-324. PMID: 4546096

8. Elwell LP, De Graaff J, Seibert D, Falkow S (1975) Plasmid-linked ampicillin resistance in Haemophilus influenzae type b. Infect Immun 12:404-410. doi: 10.1128/IAI.12.2.404-410.1975

9. Søndergaard A, Lund M, Nørskov-Lauritsen N (2015) TEM-1-encoding small plasmids impose dissimilar fitness costs on Haemophilus influenzae and Haemophilus parainfluenzae. Microbiology 161:2310-2315. doi: 10.1099/mic.0.000183

10. Trepod CM, Mott JE (2005) Elucidation of essential and nonessential genes in the Haemophilus influenzae Rd cell wall biosynthetic pathway by targeted gene disruption. Antimicrob Agents Chemother 49:824-826. doi: 10.1128/AAC.49.2.824-826.2005

11. Ubukata K, Shibasaki Y, Yamamoto K, Chiba N, Hasegawa K, Takeuchi Y, Sunakawa K, Inoue M, Konno M (2001) Association of amino acid substitutions in penicillin-binding protein 3 with $\beta$-lactam resistance in $\beta$-lactamase-negative ampicillin-resistant Haemophilus influenzae. Antimicrob Agents Chemother 45:1693-1699. doi: 10.1128/AAC.45.6.1693-1699.2001

12. Skaare D, Anthonisen IL, Kahlmeter G, Matuschek E, Natås OB, Steinbakk M, Sundsfjord A, Kristiansen BE (2014) Emergence of clonally related multidrug resistant Haemophilus influenzae with penicillin-binding protein 3-mediated resistance to extended-spectrum cephalosporins, Norway, 2006 to 2013. Euro Surveill 19(49). pii: 20986. doi: 10.2807/1560-7917.es2014.19.49.20986 
13. García-Cobos S, Campos J, Lázaro E, Román F, Cercenado E, García-Rey C, Pérez-Vázquez M, Oteo J, de Abajo F (2007) Ampicillin-resistant non-beta-lactamase-producing Haemophilus influenzae in Spain: recent emergence of clonal isolates with increased resistance to cefotaxime and cefixime. Antimicrob Agents Chemother 51:2564-2573. doi: 10.1128/AAC.00354-07

14. Deghmane $A E$, Hong $E$, Chehboub $S$, Terrade A, Falguières $M$, Sort $M$, Harrison $O$, Jolley $K A$, Taha MK (2019) High diversity of invasive Haemophilus influenzae isolates in France and the emergence of resistance to third generation cephalosporins by alteration of $f t s /$ gene. J Infect 79:7-14. doi: 10.1016/j.jinf.2019.05.007

15. Hotomi M, Fujihara K, Billal DS, Suzuki K, Nishimura T, Baba S, Yamanaka N (2007) Genetic characteristics and clonal dissemination of beta-lactamase-negative ampicillin-resistant Haemophilus influenzae strains isolated from the upper respiratory tract of patients in Japan. Antimicrob Agents Chemother 51:3969-3976. doi: 10.1128/AAC.00422-07

16. Han MS, Jung HJ, Lee HJ, Choi EH (2019) Increasing Prevalence of Group III Penicillin-Binding Protein 3 Mutations Conferring High-Level Resistance to Beta-Lactams Among Nontypeable Haemophilus influenzae Isolates from Children in Korea. Microb Drug Resist 25:567-576. doi: 10.1089/mdr.2018.0342

17. WHO Global priority list of antibiotic-resistant bacteria 2017. https://www.who.int/medicines/publications/WHO-PPL-Short_Summary_25Feb-ET_NM_WHO.pdf

18. Rebelo AR, Ibfelt T, Bortolaia V, Leekitcharoenphon P, Hansen DS, Nielsen HL, Ellermann-Eriksen S; Kemp M, Røder BL, Frimodt-Møller N, Søndergaard TS, Coia JE, Østergaard C, Pedersen M; Westh H, Aarestrup FM () One Day in Denmark: nationwide point-prevalence survey of human bacterial isolates and comparison of classical and whole-genome sequence-based species identification methods (submitted)

19. Voldstedlund M, Haarh M, Mølbak K, MiBa Board of Representatives (2014) The Danish Microbiology Database (MiBa) 2010 to 2013. Euro Surveill 19:20667. doi: 10.2807/1560-7917.es2014.19.1.20667

20. Seemann T, Goncalves da Silva A, Bulach DM, Schultz MB, Kwong JC, Howden BP. Nullarbor Github https://github.com/tseemann/nullarbor

21. Bolger AM, Lohse M, Usadel B (2014) Trimmomatic: a flexible trimmer for Illumina sequence data. Bioinformatics 30:2114-2120. doi: 10.1093/bioinformatics/btu170

22. Souvorov A, Agarwala R, Lipman DJ (2018) SKESA: strategic k-mer extension for scrupulous assemblies. Genome Biol 19:153. doi: 10.1186/s13059-018-1540-z

23. Nedergaard S, Kobel CM, Nielsen MB, Møller RT, Jensen AB, Nørskov-Lauritsen N (2019) Whole genome sequencing of Aggregatibacter actinomycetemcomitans cultured from blood stream infections reveals three major phylogenetic groups including a novel lineage expressing serotype a membrane 0 polysaccharide. Pathogens 8:256. doi: 10.3390/pathogens 8040256

24. Wattam AR, Brettin T, Davis JJ, Gerdes S, Kenyon R, Machi D, Mao C, Olson R, Overbeek R, Pusch GD, Shukla MP, Stevens R, Vonstein V, Warren A, Xia F, Yoo H (2018) Assembly, Annotation, and 
Comparative Genomics in PATRIC, the All Bacterial Bioinformatics Resource Center. Methods Mol Biol 1704:79-101. doi: 10.1007/978-1-4939-7463-4_4

25. Sinha S, Mell JC, Redfield RJ (2012) Seventeen Sxy-dependent cyclic AMP receptor protein siteregulated genes are needed for natural transformation in Haemophilus influenzae. J Bacteriol 194:5245-5254. doi: 10.1128/JB.00671-12

26. Kumar S, Stecher G, Li M, Knyaz C, Tamura K (2018) MEGA X: Molecular Evolutionary Genetics Analysis across Computing Platforms. Mol Biol Evol 35:1547-1549. doi: 10.1093/molbev/msy096

27. Meats E, Feil EJ, Stringer S, Cody AJ, Goldstein R, Kroll JS, Popovic T, Spratt BG (2003) Characterization of encapsulated and noncapsulated Haemophilus influenzae and determination of phylogenetic relationships by multilocus sequence typing. J Clin Microbiol 41:1623-1636. doi: 10.1128/jcm.41.4.1623-1636.2003

28. Søndergaard A, San Millan A, Santos-Lopez A, Nielsen SM, Gonzalez-Zorn B, Nørskov-Lauritsen N (2012) Molecular organization of small plasmids bearing blaTEM-1 and conferring resistance to $\beta$ lactams in Haemophilus influenzae. Antimicrob Agents Chemother 56:4958-4960. doi:

10.1128/AAC.00408-12

29. Hansen KH, Andreasen MR, Pedersen MS, Westh H, Jelsbak L, Schønning K (2019) Resistance to piperacillin/tazobactam in Escherichia coli resulting from extensive IS26-associated gene

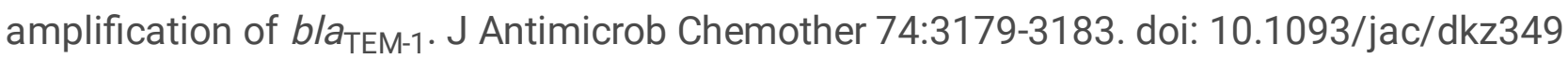

30. Honda H, Sato T, Shinagawa M, Fukushima Y, Nakajima C, Suzuki Y, Kuronuma K, Takahashi S, Takahashi H, Yokota S-I (2020) In vitro derivation of fluoroquinolone-resistant mutants from multiple lineages of Haemophilus influenzae and identification of mutations associated with fluoroquinolone resistance. Antimicrob Agents Chemother 64:e01500-19. doi: 10.1128/AAC.01500-19

31. Nørskov-Lauritsen N (2014) Classification, identification, and clinical significance of Haemophilus and Aggregatibacter species with host specificity for humans. Clin Microbiol Rev 27:214-240. doi: 10.1128/CMR.00103-13

32. Harris TM, Price EP, Sarovich DS, Nørskov-Lauritsen N, Beissbarth J, Chang AB, Smith-Vaughan HC (2020) Comparative genomic analysis identifies X-factor (haemin)-independent Haemophilus haemolyticus: a formal re-classification of 'Haemophilus intermedius'. Microb Genom 6:e000303. doi: $10.1099 /$ mgen.0.000303

33. Jorgensen JH, Ferraro MJ (2009) Antimicrobial susceptibility testing: a review of general principles and contemporary practices. Clin Infect Dis 49:1749-1755. doi: 10.1086/647952

34. Barry AL, Fuchs PC, Brown SD (2001) Identification of beta-lactamase-negative, ampicillin-resistant strains of Haemophilus influenzae with four methods and eight media. Antimicrob Agents Chemother 45:1585-1588. doi: 10.1128/AAC.45.5.1585-1588.2001

35. Skaare D, Lia A, Hannisdal A, Tveten Y, Matuschek E, Kahlmeter G, Kristiansen BE (2015) Haemophilus influenzae with Non-Beta-Lactamase-Mediated Beta-Lactam Resistance: Easy To Find but Hard To Categorize. J Clin Microbiol 53:3589-3595. doi: 10.1128/JCM.01630-15 
36. Nørskov-Lauritsen N, Ridderberg W, Erikstrup LT, Fuursted K (2011) Evaluation of disk diffusion methods to detect low-level $\beta$-lactamase-negative ampicillin-resistant Haemophilus influenzae. APMIS 119:385-392. doi: 10.1111/j.1600-0463.2011.02745.x

37. Skaare 2014b: Skaare D, Anthonisen IL, Caugant DA, Jenkins A, Steinbakk M, Strand L, Sundsfjord A, Tveten Y, Kristiansen BE (2014) Multilocus sequence typing and ftsl sequencing: a powerful tool for surveillance of penicillin-binding protein 3-mediated beta-lactam resistance in nontypeable Haemophilus influenzae. BMC Microbiol 14:131. doi: 10.1186/1471-2180-14-131

38. Maughan H, Redfield RJ (2009) Extensive variation in natural competence in Haemophilus influenzae Evolution 63(7):1852-1866. doi: 10.1111/j.1558-5646.2009.00658.x

\section{Figures}




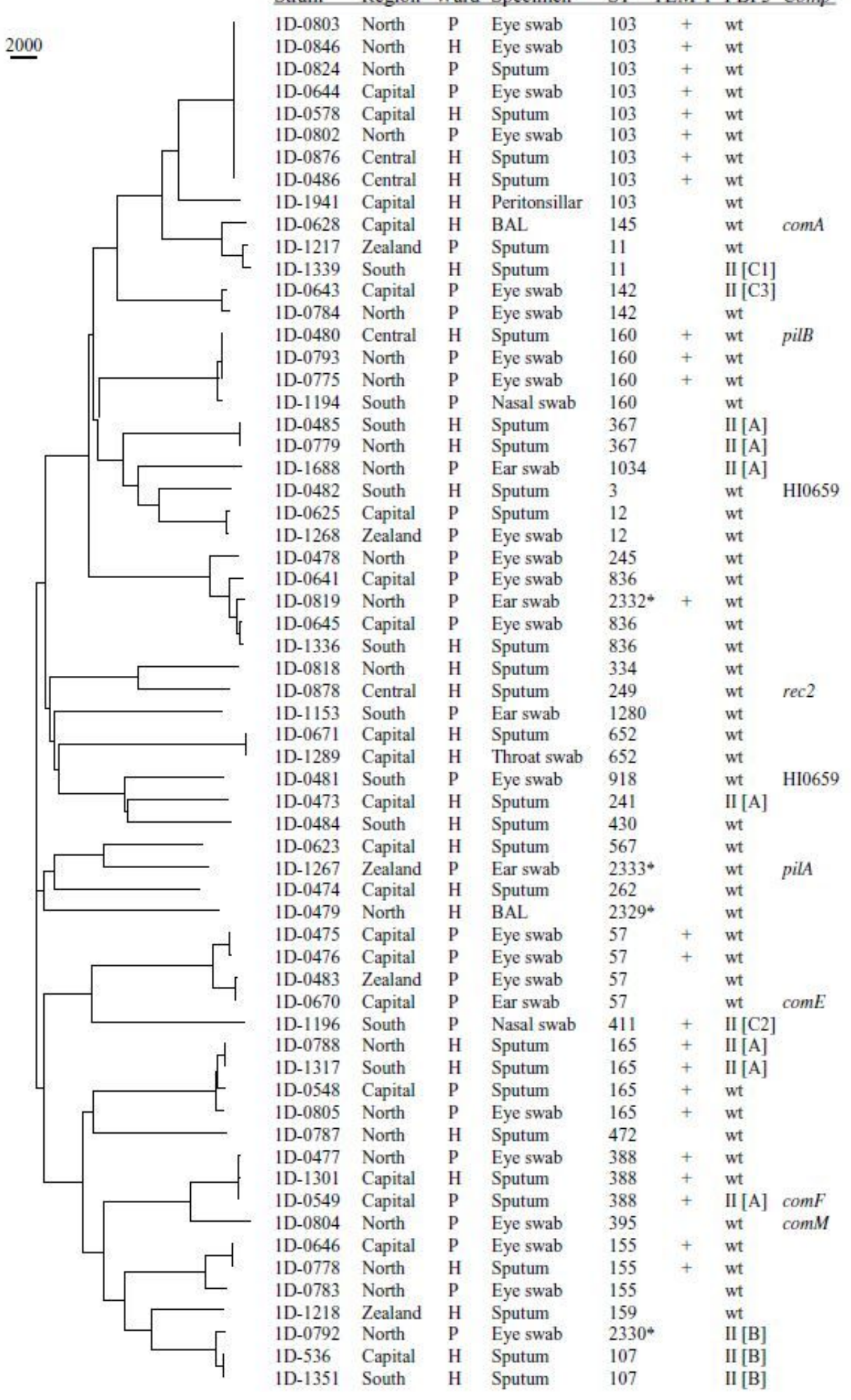

\section{Figure 1}

Neighbour-joining dendrogram of 1,166 core genes (1,136,658 nt including 103,612 positions with SNPs) of 62 isolates of $\mathrm{H}$. influenzae. Letters appended to PBP3 group II refer to ftsl clusters (Figure 4). The asterisks denote new sequence types described in this study. Bar represents 2000 residue substitutions. Abbreviations: BAL, broncho-alveolar lavage; Comp-, truncated competence genes; $\mathrm{H}$, hospital department; P, general or speciality medical practitioner; wt, wild type. 


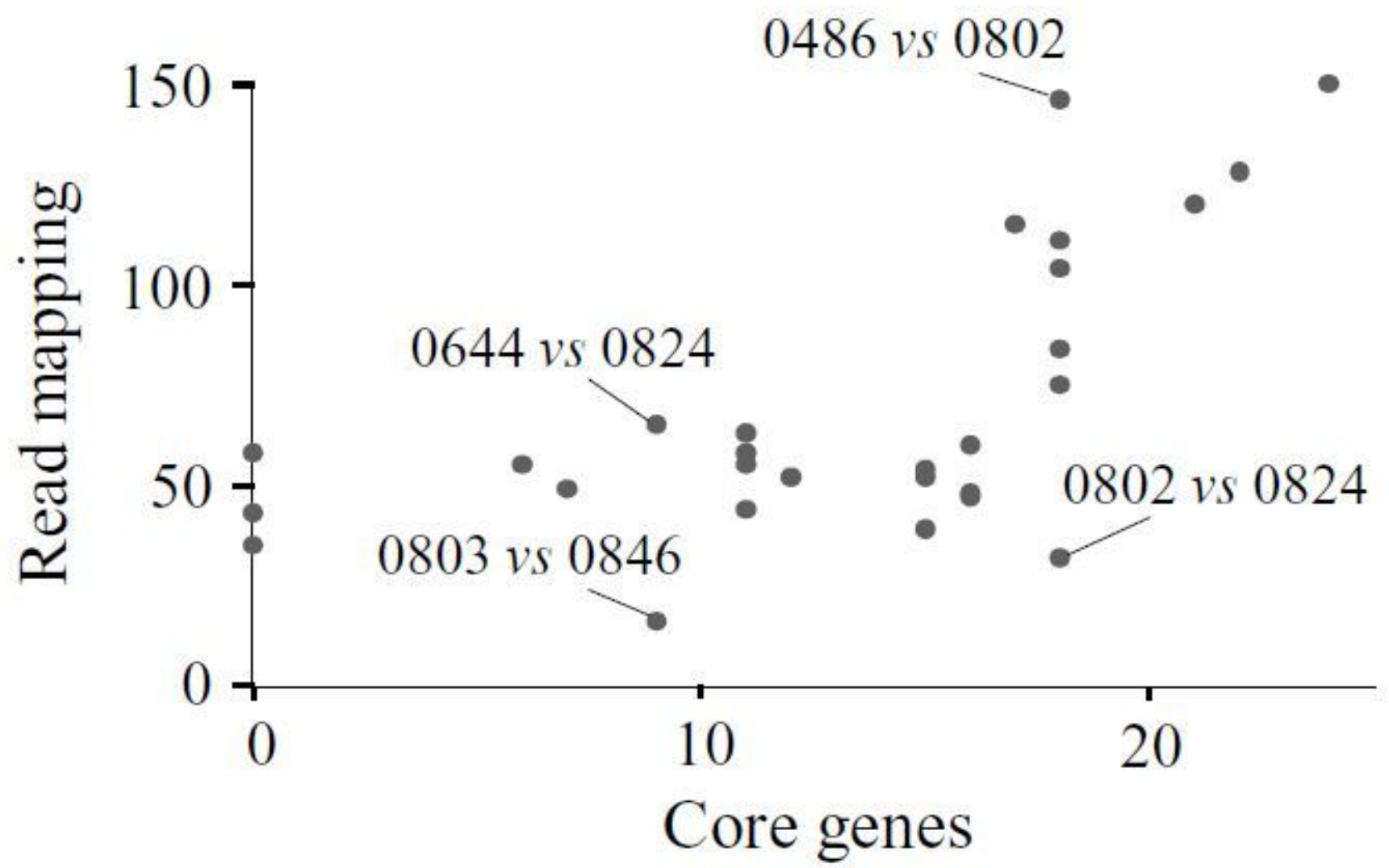

Figure 2

Scatter plot of SNP distances among closely related ST103 strains by alignment of 1,166 core genes (abscissa) and by mapping of reads to $\mathrm{H}$. influenzae strain Rd KW12 (ordinate). Selected pairs are indicated. 


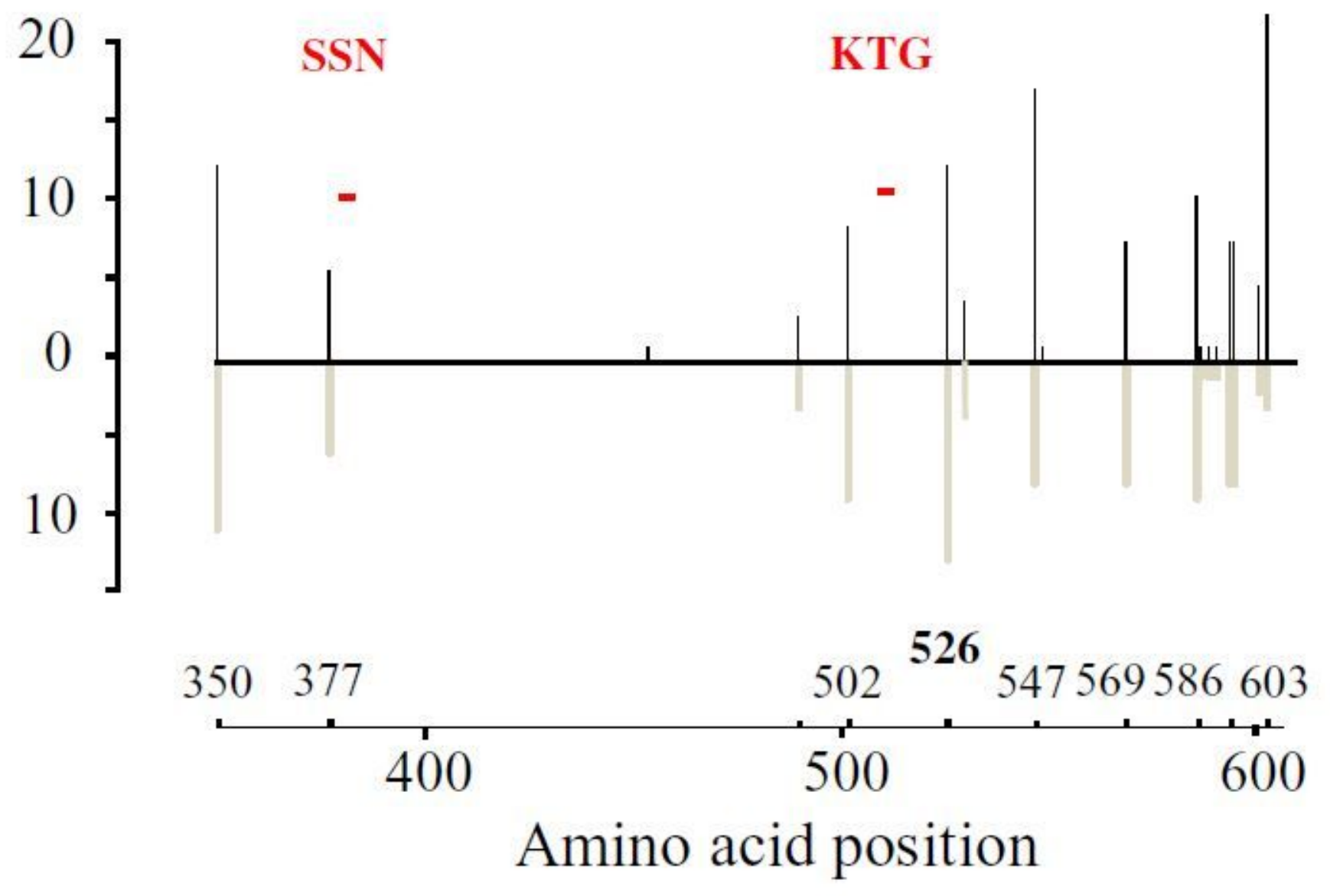

Figure 3

Substitutions in PBP3 (amino acids 350-603) using strain Rd KW20 as reference. Upward coloumns represent number of substitutions per site of 62 strains, downward coloums are restricted to 13 strains with the N526K substitution. The Ser-Ser-Asn motif at 379-381, and the Lys-Thr-Gly motif at 512-514 are indicated; the transpeptidase region spans amino acid 337-578. 


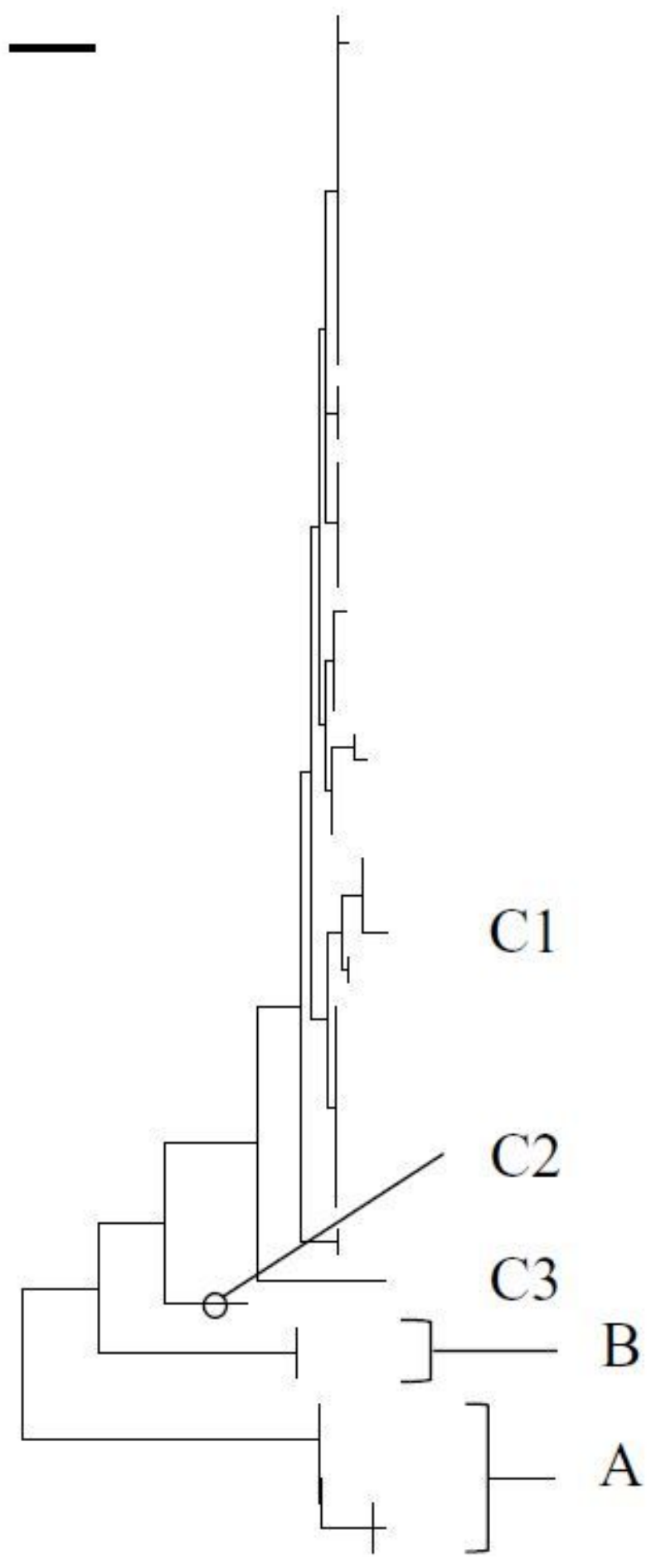

Figure 4

Neighbour-joining dendrogram of the part of $\mathrm{ftsl}$ that encodes the PBP3 transpeptidase region (nt 10081710). The 13 strains with substitution N526K cluster in two groups A and B, plus three singletons (C1C3). Bar represents seven residue substitutions. 